EDITORIAL

\title{
ACTA COLOMBIANA DE PSICOLOGÍA
}

\author{
ERNESTO RAVELO C.*
}

Se comparte con nuestros lectores que una vez actualizada la clasificación de revistas en Scimago Journal Country Rank, (SJR) nuestra revista Acta Colombiana de Psicología aparece ubicada en el cuartil 3 (Q3). Se espera que con la continua exigencia para garantizar una alta calidad científica, el aumento de la visibilidad, el logro de la cobertura, el posicionamiento y expansión geográfica de lo publicado en Acta, el fortalecimiento del sitio web de la revista, el aumento del impacto, entre otros, se contribuya a continuar alcanzando reconocimiento de las diferentes agencias internacionales que divulgan los indicadores de las publicaciones científicas en el mundo.

Así mismo, y tal como se informó en el número anterior, se continuará con la publicación de los artículos en las versiones de idioma en español e inglés y en versión del idioma portugués en los casos en que los autores envían el documento original en este idioma. En el presente volumen de Acta se publican artículos de investigadores internacionales de la Universidad Nacional Autónoma de México y la Universidad Veracruzana (México); Facultad Meridional-IMED, Universidad Federal del Santa María, Pontificia Universidad Católica del Rio Grande del Sur, Universidade São Francisco, Universidade Federal do Rio de Janeiro (Brasil); Universidad de Granada, Universidad de la Laguna (España); Universidad Tecnológica de El Salvador (El Salvador). Igualmente, artículos nacionales de la Universidad Católica de Colombia, Universidad de los Andes, Bogotá y Empresa de consultoría Prax S.A.S.

Las áreas de la Psicología que se tienen en cuenta en los artículos presentados corresponden al área de Salud, Experimental, Clínica, Educativa, Neuropsicología, Social, Gerontología, Bibliometría y Psicometría. En cuanto a los temas tratados, Serrano, Sosa y González presentan los resultados de la investigación en donde el objetivo fue evaluar el desarrollo del control del estímulo bajo un programa de reforzamiento definido temporalmente, encontrando que la disponibilidad limitada de reforzamiento de los programas

\footnotetext{
Editor

revistaacta@ucatolica.edu.co
}

definidos temporalmente no impide el control del estímulo, así como que dicho control se desarrolla sin patrones conductuales derivados de la regularidad secuencial entre los subciclos. Cantor y Avendaño llevan a cabo un estudio para determinar las propiedades psicométricas del test de cribado de demencias Pesotest en muestras clínica y no clínica de adultos mayores de la ciudad de Bogotá, Colombia. Oliveira, Pagliarin, Calvette Bastos y Fonseca, investigan el procesamiento comunicativo de pacientes con accidente cerebrovascular (ACV) de hemisferio derecho; Noronha y Martins determinan las relaciones entre fuerzas de carácter y satisfacción con la vida, así como las diferencias de media entre sexo y edad en estudiantes universitarios. Por su parte, Navarro-Mantas y Velásquez proponen una herramienta de registro de situaciones cotidianas de desigualdad de género que fue empleada en un programa de prevención de violencia de género cuya evaluación se hizo a través de un diseño cuasi-experimental. En su investigación, López y Argimon caracterizan a ancianos que participaron en un entrenamiento cognitivo, con la finalidad de medir los efectos del entrenamiento, con énfasis en las funciones ejecutivas, comparando un grupo experimental con un grupo control. De acuerdo con la Teoría de la Restauración, Mejía-Castillo, López-Suárez, Estrada y Lagunes-Córdoba presentan su estudio con estudiantes de centros educativos de nivel de bachillerato en Xalapa, México, para identificar y comparar las cualidades restauradoras percibidas en sus espacios escolares. La investigación de Gómez, Segura, Castrillón y Perilla tuvo como objetivo construir y validar una batería que supere algunas de las limitaciones que se han encontrado con la batería de factores de Riesgo Psicosocial del Ministerio de la Protección Social en Colombia. Con el propósito de evaluar la efectividad de un programa de intervención para promover la salud sexual de adolescentes que se encuentran en conflicto con la ley se presenta al aporte del estudio de Sánchez, Enríquez y Robles. Por otra parte, Ravelo, Mejía y González realizan un análisis descriptivo de los artículos publicados en la Revista Acta Colombiana de Psicología entre 2010 y 2014. Por último, el objetivo del aporte de Londoño y González fue evaluar la prevalencia de depresión, detectar el riesgo suicida e identificar los factores sociodemográficos y personales 
asociados a este trastorno, en una muestra de hombres de nacionalidad colombiana.

Agradecemos a todos los autores de los artículos, a los grupos y redes de investigación, a los árbitros, a los miembros del Comité Editorial, del Comité Científico, del Consejo Editorial, y al equipo de traductores, revisores metodológicos y estadísticos, técnicos y de estilo, por contribuir al buen nivel académico y científico de Acta Colombiana de Psicología. 